\title{
High Precision Edge Detection Algorithm for Mechanical Parts
}

\author{
Zhenyun Duan ${ }^{1}$, Ning Wang ${ }^{1}$, Jingshun $\mathrm{Fu}^{1}$, Wenhui Zhao ${ }^{1}$, Boqiang Duan ${ }^{2}$, Jungui Zhao ${ }^{3}$ \\ ${ }^{1}$ School of Mechanical Engineering, Shenyang University of Technology, Shenyang, 110870, People's Republic of China \\ ${ }^{2}$ College of Aerospace Engineering, Nanjing University of Aeronautics and Astronautics, Nanjing, 210016, \\ People's Republic of China \\ ${ }^{3}$ Development Planning Department, China Academy of Launch Vehicle Technology, Beijing, 100076, \\ People’s Republic of China,fjs_sut@126.com
}

\begin{abstract}
High precision and high efficiency measurement is becoming an imperative requirement for a lot of mechanical parts. So in this study, a subpixel-level edge detection algorithm based on the Gaussian integral model is proposed. For this purpose, the step edge normal section line Gaussian integral model of the backlight image is constructed, combined with the point spread function and the single step model. Then gray value of discrete points on the normal section line of pixel edge is calculated by surface interpolation, and the coordinate as well as gray information affected by noise is fitted in accordance with the Gaussian integral model. Therefore, a precise location of a subpixel edge was determined by searching the mean point. Finally, a gear tooth was measured by M\&M3525 gear measurement center to verify the proposed algorithm. The theoretical analysis and experimental results show that the local edge fluctuation is reduced effectively by the proposed method in comparison with the existing subpixel edge detection algorithms. The subpixel edge location accuracy and computation speed are improved. And the maximum error of gear tooth profile total deviation is $1.9 \mu \mathrm{m}$ compared with measurement result with gear measurement center. It indicates that the method has high reliability to meet the requirement of high precision measurement.
\end{abstract}

Keywords: Vision measurement, edge detection, subpixel-level, Gaussian integral model, normal section line.

\section{INTRODUCTION}

Mechanical parts are widely used in most major equipment. The high precision and high efficiency measurement for these components is becoming more and more urgent. With the rapid development of non-contact, high precision and automation computer vision technology, the computer vision and its applications become a research hotspot. Vision measurement can quickly and efficiently detect the contours and plane shapes, angles and positions of various complex parts, especially the microscopic detection and quality control of precision components, which achieves the rapid measurement of object size or relative position [1]-[4].

Recently, vision measurement has gradually attracted attention and applications [5]-[6]. How to realize the edge detection is one of key technologies in vision measurement because it decides the measuring accuracy. In order to shift the measuring accuracy, one method is improving the accuracy of measuring equipment, which will increase the measuring expense. Another proper method is subpixel-level measurement. According to current mathematical models, subpixel detection methods can mainly be divided into three types, the moment method, fitting method, and interpolation method [7]-[11]. Among these methods, fitting method has higher positioning accuracy owing to that it can filter off noise pixels. Thus, as a fitting method, the Facet surface model has been widely used as an image model. This model fits the gray surface with the gray information of discrete digital image to determine the subpixel edge location [12][14]. One problem of this model is that it uses all pixels in a symmetrical area with the edge pixel points around, including the image background and foreground, and a few pixel points in transitional zone. So, it may own a large error in the extraction of subpixel edge and low computing efficiency.

Therefore, this study proposes an accurate and efficient subpixel edge detection algorithm based on the Gaussian integral model to realize the high precision measurement of the mechanical parts, such as gauge block and standard involute gear.

\section{EDGE CHARACTERISTICS}

Step edge and roof edge are two types of common image edges. Edge of backlight image belongs to step edge. So this study focuses on step edge.

The actual lens imaging system is limited by the modulation transfer function (MTF) cutoff frequency. Actual image can be considered as the convolution of imaging point spread function (PSF) and the ideal image 
function. The actual edge as shown in Fig.1.a), and the first derivative distribution of actual edge is consistent with Gaussian distribution as shown in Fig.1.b) [15]. It is the same with the point spread function of the imaging system.

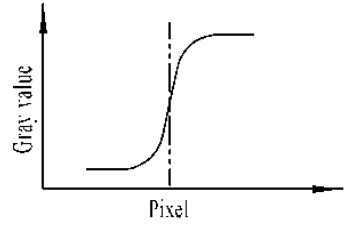

a) Actual edge

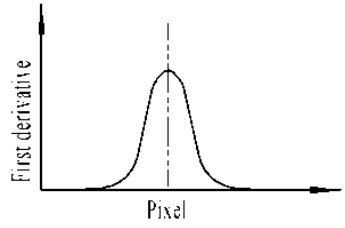

b) First derivative
Fig.1. Gray distribution of step edge.

In this study, charge-coupled device (CCD) was used to capture image. According to the square aperture sampling principle, the gray value is equal to the integration of CCD light intensity with the fixed area in a fixed time period. The output results are the pixel gray value of image, which can be expressed as a discrete matrix [16].

\section{DETECTION METHOD OF PIXEL EDGE}

\section{A. Image smoothing}

In the collecting and transferring process of image, some noise is usually produced. It influences the subsequent processing results seriously. So it is important to preprocess the original image to reduce noise after image capture. Image smoothing is a practical digital image processing technology to reduce the noise. A better smoothing method usually eliminates image noise and also guarantees clear image edge contour.

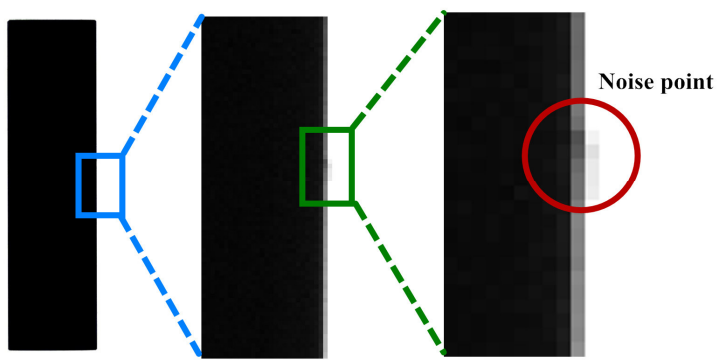

a) Original image

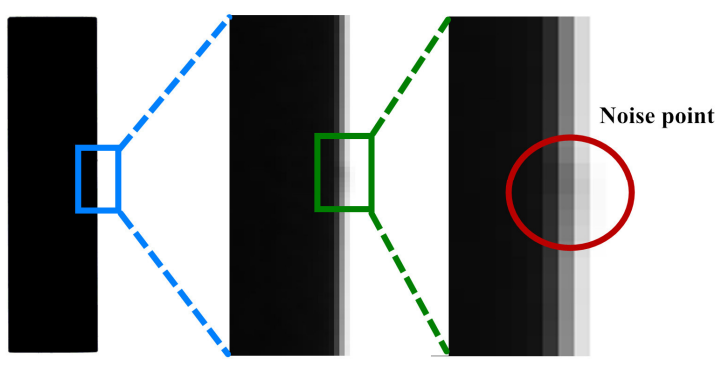

b) Gaussian filter processed image

Fig.2. Comparison between original and Gaussian filter processed image.
The most noise signals of image are according to Gaussian distribution. So the Gaussian filter could be an effective method to suppress noise.

One dimensional Gaussian function $\mathrm{G}(\mathrm{x})$ was chosen to establish the filter. Basing on the convolution processing of original image $f(i, j)$, the gray value of smooth image $I(i, j)$ can be expressed as

$$
I(i, j)=[G(i) G(j)]^{*} f(i, j)
$$

Fig.2. shows the original and Gaussian filter processed image. It can be seen that Gaussian filter removes the noise points in the marginal zone. It increases the gray contrast.

\section{B. Edge detection process}

In digital image, each target pixel has eight neighborhood pixels except for the boundary one. It is very complex for traditional detection algorithm to calculate first or second order derivative, so as to determine the image edge by means of pixel gray tendency. If the neighborhood pixels are only considered and are not calculated repeatedly, the processing time will be reduced effectively. Therefore, here an eight-neighborhood edge tracking algorithm is proposed to realize edge detection. The process to detect pixel edge is as follows:

1) The gray value of each pixel is first scanned by column. If the gray value of a point $f(i, j) \geq T$, then this point $\left(x_{i}, y_{i}\right)$ is considered as the edge of the starting point, its coordinate is noted as tag to avoid repeating track. As shown in Fig.3., this point is marked as starting point $\mathrm{P} 0$.

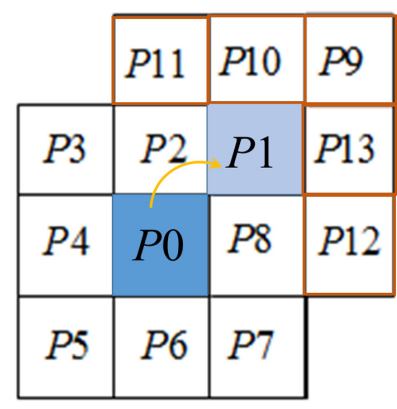

Fig.3. Schematic diagram of edge tracking.

2) After P0 is considered as the starting point of the edge, the maximum gray value point is searched around the eight neighborhoods of the point $\mathrm{P} 0$. The maximum gray value point is considered as the next edge point of the image, named as P1. After that the point P1 is marked as the next edge point. According to the two existing edge points $\mathrm{P} 0, \mathrm{P} 1$ and characteristics of single pixel edge, the next maximum gray value point after $\mathrm{P} 1$ cannot be $\mathrm{P} 2$ and $\mathrm{P} 8$. So the gray value of these two points is cleared. When searching the maximum gray value point around the eight neighborhoods of the point $\mathrm{P} 1$, the maximum point is only one of the P9 to P13. So, these points can only be calculated, which greatly reduces the computing complexity. 
3) When the searching point is located at the boundary or starting point of the image, the edge extraction is finished, else repeat the process.

\section{SUBPIXEL EDGE DETECTION}

Subpixel edge point is located in the normal direction of the edge section line. In order to determine accurate position, Gaussian integral model is established to obtain the location of subpixel edge by searching the mean point.

\section{A. Establishing the Gaussian integral model}

According to the above analysis, the PSF [17] can be expressed by

$$
p(t)=\frac{1}{\sqrt{2 \pi} \sigma} e^{-\frac{(t-u)^{2}}{2 \sigma^{2}}}
$$

where $t$ denotes the impacted point coordinate, $u$ is the spread point coordinate, and $\sigma$ is the standard deviation.

The ideal step edge can be defined as

$$
E(t)= \begin{cases}g & t \leq \mu \\ g+k & t>\mu\end{cases}
$$

where $\mu$ denotes edge, and $k$ is the gray values difference between the background and prospect.

The theoretical gray value of an image can be expressed as follows:

$$
\begin{aligned}
& P(t)=\int_{-\infty}^{+\infty} p(t) E(t) \\
& =\int_{-\infty}^{\mu} p(t) E(t)+\int_{\mu}^{+\infty} p(t) E(t) \\
& =g+\frac{k}{\sqrt{2 \pi} \sigma} \int_{-\infty}^{\mu} e^{-\frac{(t-u)^{2}}{2 \sigma^{2}}} d t
\end{aligned}
$$

It is difficult to solve the integral expression directly. In order to obtain the mean point of the Gaussian integral model, the $(t-u) / \sigma$ was replaced by $v$. Therefore,

$$
\begin{aligned}
& P(t)=g+\frac{k}{\sqrt{2 \pi}} \int_{-\infty}^{\frac{t-\mu}{\sigma}} e^{-\frac{v^{2}}{2}} d v \\
& =g+k \phi(v)=g+k \phi\left(\frac{t-\mu}{\sigma}\right)
\end{aligned}
$$

According to the standard normal distribution table, the upper limit of the integral in (5) can be defined as $a_{i}=t_{i}-\mu / \sigma$.

According to the minimum mean square error principle of least-squares fitting, we can obtain

$$
\sum_{i=-N}^{N}\left(t_{i}-\mu-a_{i} \sigma\right)^{2}=C
$$

Then, the partial derivative of $C$ to $\mu$ and $\sigma$ is set equal to zero.

$$
\left\{\begin{array}{l}
\frac{\partial C}{\partial \mu}=2 \sum_{i=-N}^{N}\left(t_{i}-\mu-a_{i} \sigma\right)=0 \\
\frac{\partial C}{\partial \sigma}=2 \sum_{i=-N}^{N}\left(a_{i} t_{i}-a_{i} \mu-a_{i}^{2} \sigma\right)=0
\end{array}\right.
$$

This can be further solved to obtain

$$
\left\{\begin{array}{l}
\mu=\bar{t}_{i}-\sigma \cdot \frac{\overline{t_{i}} \cdot \overline{a_{i}}-\overline{t_{i} a_{i}}}{\overline{a_{i}} \cdot \overline{a_{i}}-\overline{a_{i}^{2}}} \\
\sigma=\frac{\overline{t_{i}} \cdot \overline{a_{i}}-\overline{t_{i} a_{i}}}{\overline{a_{i}} \cdot \overline{a_{i}}-\overline{a_{i}^{2}}}
\end{array}\right.
$$

Where $\overline{t_{i}}, \overline{a_{i}}, \overline{t_{i} a_{i}}$, and $\overline{a_{i}^{2}}$ denote the arithmetic mean value of $t_{i}, a_{i}, t_{i} a_{i}, a_{i}^{2}$, respectively.

$\mu$ denotes the distance between subpixel edge points in normal section line and pixel edge points, $t$ denotes the distance between discrete points on the normal section line and pixel edge points. As shown in Fig.4., the coordinates of subpixel edge points can be easily obtained.

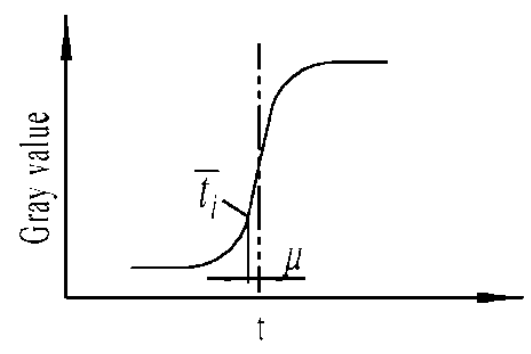

Fig.4. Gaussian integral model on normal section line.

\section{B. Solution method for the proposed model}

The subpixel edge information could be obtained by solving the above Gaussian integral model. The solving process to locate the subpixel edge involves:

1) The pixel edge of image was first thrice fitted with the least-squares method. Then the pixel edge points were obtained by discretizing the fitting curve. The normal section line of each pixel edge point was also determined based on the fitting curve.

2) The normal offset curve could be obtained on each side of the pixel edge-fitting curve by choosing a series of equidistant lines in arithmetic progression. Then the Gaussian integral model fitting points can be determined by finding the intersections of the normal section line and the normal offset curve.

3) The gray value of fitting points was given to the Gaussian integral model by using Bezier surface interpolation. Then, the initial gray value of fitting point was filtered along the tangential direction of the normal offset 
curve with the Gaussian filter method. Lastly, the final gray value of the fitting points was obtained.

4) The gray values of discrete points in normal section line of pixel edge were fitted based on Gaussian integral model. Then the coordinates of subpixel edge were obtained according to the mean point $\mu$ of the Gaussian integral model using (8).

\section{EXPERIMENT}

The vision measurement system was designed as shown in Fig.5. It mainly consists of a CCD camera, double telecentric lens, LED blue light, digital controller, guide rail slider and holder. It can be used for the measurement of mechanical parts.

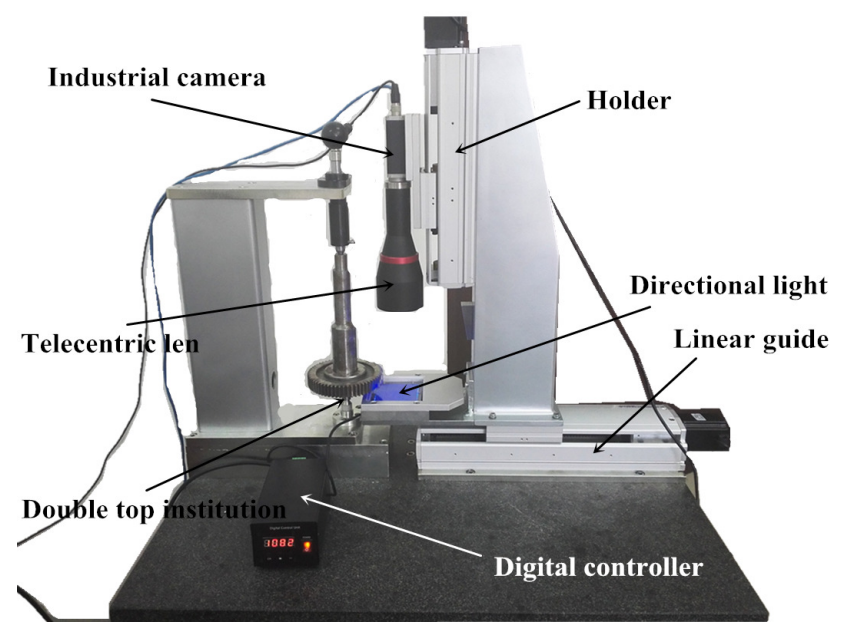

Fig.5. Vision measurement system.

\section{A. Error analysis of subpixel location}

According to the JJG2056-90 standard verification system for length measuring instruments, the accuracy of the firstgrade gauge block with the size of $1 \mathrm{~mm}$ to $10 \mathrm{~mm}$ is below $0.05 \mu \mathrm{m}$. Due to its high accuracy and simple edge, the firstgrade gauge block with the size of $5 \mathrm{~mm}$ in different positions was used for verification of the above algorithm. Fig.6. shows the images of the gauge block image with the size of $5 \mathrm{~mm}$ in different positions. In order to analyze the accuracy of the algorithm, the proposed algorithm and Facet surface fitting method were compared to detect the subpixel edge of the gauge block.

Owing to the high quality of the gauge block edge, the result of each image processing shows the same character. It illustrates that the position of image has no effect on the image processing. Therefore, the lower edge of the gauge block was taken, for example, to verify the proposed algorithm as shown in Fig.7. First, seven normal offset curves on each side of the pixel edge fitting curve were obtained symmetrically, each interval between equidistant lines can be expressed by arithmetic progression, of which the first term is 0.3 and the common difference is 0.1 . Thus, fifteen discrete points on the pixel edge normal section line were chosen for fitting. For example, certain point coordinate on the fitting pixel edge is $(1289.994,1253.711)$, its normal direction angle is $2.94^{\circ}$. The discrete points information corresponding to this point is provided in Table 1., and the Gaussian integral fitting curve is shown in Fig. 8 .
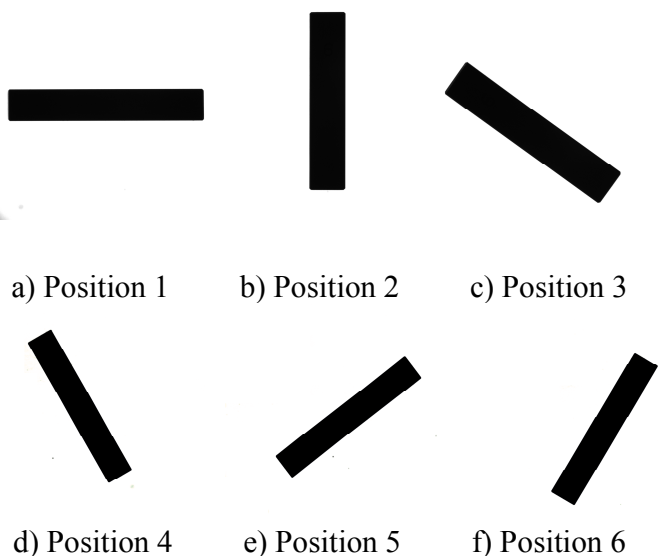

Fig.6. Gauge block image.

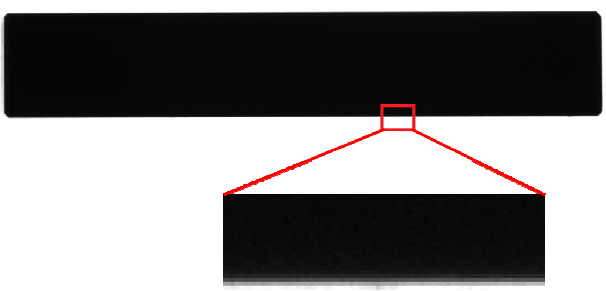

Fig.7. Edge image for vision measurement.

Table 1. Information of discrete points on normal section line of pixel edge.

\begin{tabular}{|c|c|c|c|}
\hline No. & $t /$ pixel & $\begin{array}{c}\text { Gray } \\
\text { value }\end{array}$ & $\begin{array}{c}\text { Image } \\
\text { coordinates/pixel }\end{array}$ \\
\hline 1 & -4.2 & 14.384 & $(1290.204,1249.516)$ \\
\hline 2 & -3.3 & 17.112 & $(1290.159,1250.415)$ \\
\hline 3 & -2.5 & 24.166 & $(1290.119,1251.214)$ \\
\hline 4 & -1.8 & 37.819 & $(1290.084,1251.913)$ \\
\hline 5 & -1.2 & 57.367 & $(1290.054,1252.512)$ \\
\hline 6 & -0.7 & 80.266 & $(1290.029,1253.012)$ \\
\hline 7 & -0.3 & 102.108 & $(1290.007,1253.411)$ \\
\hline 8 & 0 & 119.600 & $(1289.994,1253.711)$ \\
\hline 9 & 0.3 & 137.851 & $(1289.978,1254.010)$ \\
\hline 10 & 0.7 & 162.514 & $(1289.958,1254.410)$ \\
\hline 11 & 1.2 & 190.083 & $(1289.933,1254.909)$ \\
\hline 12 & 1.8 & 217.823 & $(1289.903,1255.508)$ \\
\hline 13 & 2.5 & 238.706 & $(1289.868,1256.208)$ \\
\hline 14 & 3.3 & 254.586 & $(1289.828,1257.007)$ \\
\hline 15 & 4.2 & 254.998 & $(1289.783,1257.905)$ \\
\hline
\end{tabular}

The mean value of the Gaussian integral fitting curve is 0.117 pixels. According to the coordinates of pixel edge and normal direction angle, the corresponding coordinate of the subpixel edge is $(1289.988,1253.828)$. 
Fig.9. shows the subpixel detection results obtained by the proposed algorithm and Facet surface fitting algorithm. It concludes that the extracted subpixel in the proposed algorithm is consistent with the Facet surface fitting algorithm, which verifies the proposed algorithm. Fig.10. gives the comparison of the fitted subpixel of the two-edge detection algorithm. As can be seen from the figure, straightness error of local fluctuation in the subpixel edge extracted by the Facet surface fitting algorithm is $6 \mu \mathrm{m}$. The straightness of local fluctuation in the subpixel edge is smoother when using the proposed algorithm, with error of $1 \mu \mathrm{m}$.

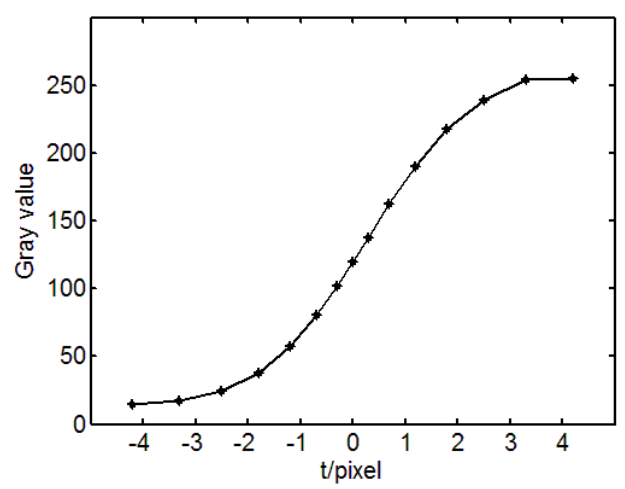

Fig.8. Gaussian integral fitting curve on normal section line.

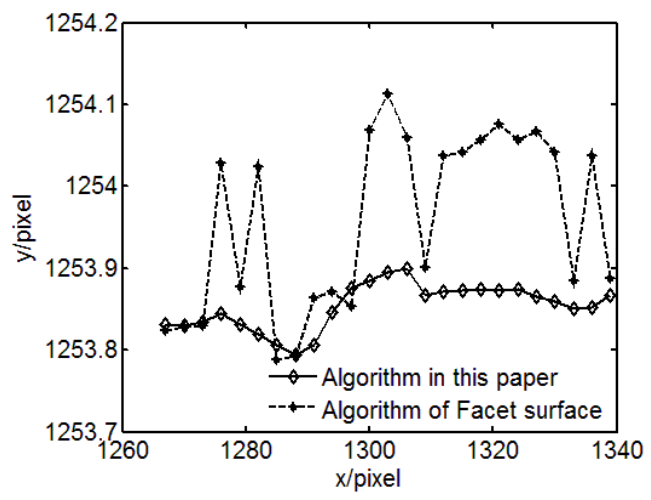

Fig.9. Subpixel edge obtained by the two algorithms.

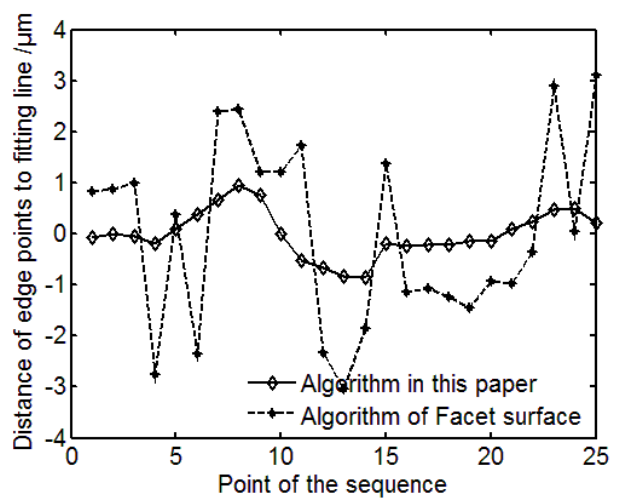

Fig.10. Distance of pixel edge points to the fitting line.
The $5 \mathrm{~mm}$ gauge block in a different position was measured to verify the measuring accuracy of this algorithm. Then the subpixel edge of the gauge block was extracted using the two algorithms proposed in this paper and the Facet surface fitting algorithm. One-gauge block edge was first fitted using the least-squares method and then the distance between the points on the other edge and the fitting line was calculated. In that case the measuring size of gauge block can be expressed by the average distance. Then, the measuring error was obtained by comparing the theoretical size with the measured size. The execution time for the proposed algorithm and that of the Facet surface fitting algorithm are $29 \mathrm{~ms}$ and $47 \mathrm{~ms}$, respectively. The measuring errors of two algorithms are provided in Table 2. The calculated results indicate that the subpixel edge extraction method of the proposed algorithm is reliable. It generates a smaller measuring error than that of the Facet surface fitting algorithm.

Table 2. Measuring error of two algorithms $(\mu \mathrm{m})$.

\begin{tabular}{|c|c|c|}
\hline $\begin{array}{c}\text { Measuring } \\
\text { image }\end{array}$ & $\begin{array}{c}\text { Proposed } \\
\text { Algorithm }\end{array}$ & $\begin{array}{c}\text { Algorithm for } \\
\text { Facet surface } \\
\text { fitting }\end{array}$ \\
\hline Position 1 & 0.914 & 1.945 \\
\hline Position 2 & 0.887 & 1.624 \\
\hline Position 3 & 0.983 & 1.817 \\
\hline Position 4 & 0.873 & 1.778 \\
\hline Position 5 & 0.941 & 1.921 \\
\hline Position 6 & 1.183 & 1.883 \\
\hline Average & 0.964 & 1.828 \\
\hline
\end{tabular}

\section{B. Verification using high precision gear}

The built-in vision measurement system was calibrated by our previous work [18]. Thus, it can be used to measure tooth profile total deviation of gear. The measured gears are standard involute spur gear. The basic parameters are shown in Table 3.

Table 3. Basic parameters of the measured gear.

\begin{tabular}{cccc}
\hline Order & $\begin{array}{c}\text { Tooth } \\
\text { number }\end{array}$ & Modulus & $\begin{array}{c}\text { Grade } \\
\text { level }\end{array}$ \\
\hline Gear \#1 & 90 & 2 & 5 \\
Gear \#2 & 60 & 2 & 5 \\
\hline
\end{tabular}

On the basis of obtaining pixel edge of gear profile, each profile was fitted with involute line. Then accurately locating subpixel profile was achieved by the section 4 algorithm. According to the coordinate of gear center [19], the initial phase angle $\varphi$ i corresponding to each point of involute tooth profile can be obtained. The gear radius of base circle $r_{b}$ has been known. So, the tooth profile total deviation of gear can be indicated by using the involute initial phase angle,

$$
\varepsilon=r_{b} \times\left|\max \left(\varphi_{j}\right)-\min \left(\varphi_{j}\right)\right|
$$


In order to analyze the accuracy of the measurement method in this paper, the M\&M3525 gear measurement center was adopted as shown in Fig.11. The tooth profile total deviation of the gear with the proposed method was compared with the measurement result of gear measurement center.

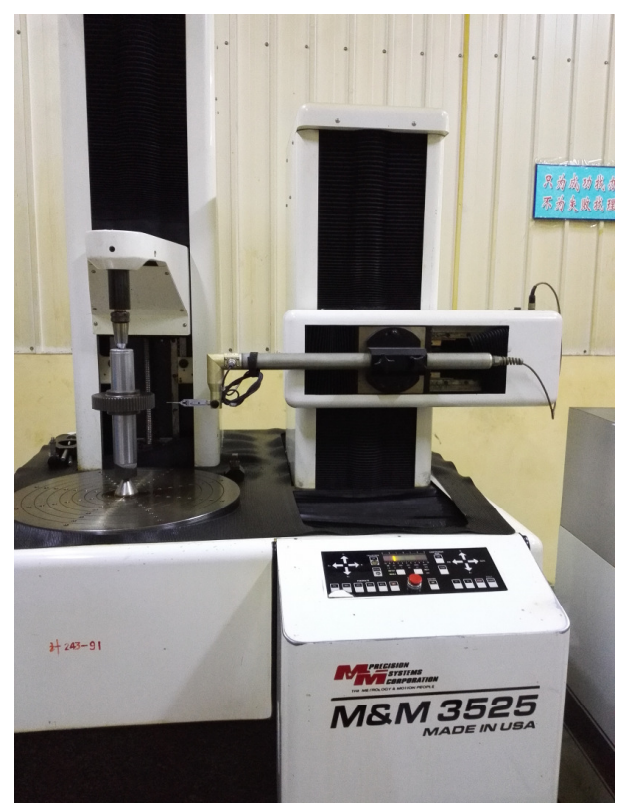

Fig.11. Gear measurement center.

The measured tooth profile total deviation obtained by two methods is shown in Table 4. M1 is the measurement result of the proposed method. M2 is the measurement result obtained by the gear measurement center. The $\Delta M$ is the difference between them. The biggest error of the two methods is smaller than $1.9 \mu \mathrm{m}$. And the trend of measurement results is basically the same. It shows that the method in this paper has certain reliability to high accuracy of measurement.

Table 4. Measurement result of two methods $(\mu \mathrm{m})$.

\begin{tabular}{|c|c|c|c|c|c|c|c|}
\hline \multirow{2}{*}{\multicolumn{2}{|c|}{$\begin{array}{l}\text { Measured } \\
\text { tooth }\end{array}$}} & \multicolumn{3}{|c|}{ Gear \#1 } & \multicolumn{3}{|c|}{ Gear \#2 } \\
\hline & & \multirow{2}{*}{$\begin{array}{l}\boldsymbol{M}_{1} \\
5.2 \\
\end{array}$} & \multirow{2}{*}{$\begin{array}{l}\boldsymbol{M}_{2} \\
4.8 \\
\end{array}$} & \multirow{2}{*}{$\frac{\Delta M}{0.4}$} & \multirow{2}{*}{$\begin{array}{l}M_{1} \\
4.3 \\
\end{array}$} & \multirow{2}{*}{$\begin{array}{l}\boldsymbol{M}_{2} \\
3.2 \\
\end{array}$} & \multirow{2}{*}{$\begin{array}{c}\boldsymbol{\Delta M} \\
0.9 \\
\end{array}$} \\
\hline Tooth & Left & & & & & & \\
\hline 1 & Right & 5.1 & 3.6 & 1.5 & 3.4 & 1.7 & 1.7 \\
\hline \multirow{2}{*}{$\begin{array}{c}\text { Tooth } \\
2 \\
\end{array}$} & Left & 5.7 & 5.2 & 0.5 & 3.9 & 2.1 & 1.8 \\
\hline & Right & 4.0 & 3.6 & 0.4 & 4.5 & 3.6 & 0.9 \\
\hline \multirow{2}{*}{\begin{tabular}{|c|} 
Tooth \\
$\mathbf{3}$ \\
\end{tabular}} & Left & 5.9 & 4.7 & 1.2 & 4.1 & 3.3 & 0.8 \\
\hline & Right & 3.5 & 2.7 & 0.8 & 5.4 & 3.5 & 1.9 \\
\hline \multirow{2}{*}{$\begin{array}{c}\text { Tooth } \\
4 \\
\end{array}$} & Left & 5.1 & 4.2 & 0.9 & 5.3 & 3.6 & 1.7 \\
\hline & Right & 6.3 & 4.9 & 1.4 & 5.4 & 3.8 & 1.6 \\
\hline
\end{tabular}

According to the relevant provisions of GB/T 10095, the tooth profile total deviation allowable value of two fifth level spur gear is $7 \mu \mathrm{m}$ and $6 \mu \mathrm{m}$. The measured results show that the tooth profile total deviation obtained by two methods is less than the maximum. We can conclude that the gears meet the accuracy requirement of fifth level gear.

\section{CONCLUSIONS}

A subpixel edge detection algorithm based on the Gaussian integral model was proposed. The algorithm constructed the step edge normal section Gaussian integral model. Based on the obtained normal of the pixel edgefitting curve, a Gaussian filter along the tangential of the edge was applied. The gray value of discrete points on the normal section line of the pixel edge was calculated with surface interpolation. This maintains the smoothness of the edge tangential and the steepness of the edge normal. Coordinate and gray information was fitted in accordance with the Gaussian integral model for accurate subpixel location. This approach solved the problem of sensitivity to gray value change by using a gradient to determine the subpixel edge; thus, it has good noise resistance, and it improves the precision of edge detection. In addition, the algorithm adopts curve fitting, reduces the amount of calculation, and improves the calculation speed compared to the Facet surface fitting.

The built-in vision measurement system was calibrated, then, the algorithm was applied to measure the gauge block and standard involute spur gear. The measurement error of first grade gauge block is $0.964 \mu \mathrm{m}$, and the tooth profile total deviation measured by this method is compared with the measurement result of the gear measurement center, its maximum error is $1.9 \mu \mathrm{m}$, which indicates that the method has high reliability and can meet the requirement of high precision measurement.

\section{ACKNOWLEDGMENT}

This work was supported by Funder: Key Projects in the National Science \& Technology Pillar Program No.2014BAF08B01.

\section{REFERENCES}

[1] Kumar, B.M, Ratnam, M.M. (2015). Machine vision method for non-contact measurement of surface roughness of a rotating workpiece. Sensor Review, 35 (1), 10-19.

[2] Gadelmawla, E.S. (2011). Computer vision algorithms for measurement and inspection of spur gears. Measurement, 44 (9), 1669-1678.

[3] Kosarevsky, S., Latypov, V. (2013). Detection of screw threads in computed tomography 3D density fields. Measurement Science Review, 17 (2), 93-99.

[4] Robinson, M.J., Oakley, J.P., Cunningham, M.J. (1995). The accuracy of image analysis methods in spur gear metrology. Measurement Science and Technology, 6, 860-871.

[5] Chen, F., Brown, G.M. (2000). Overview of threedimensional shape measurement using optical methods. Optical Engineering, 39 (1), 10-21.

[6] Lu, N.-G., Deng, W.-Y., Wang, Y.-Q. (2005). Profile measurement of microwave antenna using close range photogrammetry. In Third International Conference on Experimental Mechanics and Third Conference of the Asian Committee on Experimental Mechanics, Proc. SPIE 5852, 508-515. 
[7] Sidor, K., Szlachta, A. (2017). The impact of the implementation of edge detection methods on the accuracy of automatic voltage reading. Measurement Science Review, 13 (6), 292-297.

[8] Shang, Y.-C., Chen, J., Tian, J.-W. (2010). The study of sub-pixel edge detection algorithm based on the function curve fitting. In 2nd International Conference on Information Engineering and Computer Science. IEEE, 1-4.

[9] Wei, B.-Z., Zhao, Z.-M. (2013). A sub-pixel edge detection algorithm based on Zernike moments. The Imaging Science Journal, 61, 436-446.

[10] Liu, G., Liu, B., Chen, F., Hu, T. (2009). Study on the method of the accuracy evaluation of sub-pixel location operators. Acta Optica Sinica, 29, 3446-3451.

[11] Li, S., Lu, R., Shi, Y. et al. (2011). Sub-pixel edge detection algorithm based on Gaussian Surface Fitting. Tool Engineer, 45, 79-82.

[12] Ma, R., Zeng, L., Lu, Y. (2009). Improved sub-pixel edge detection based on Facet model. Journal of Basic Science and Engineering, 17, 296-302.

[13] Wang, K., Zhang, D., Huang, H. et al., (2005). A study of sub voxel edge detection method based on 3-D Facet model. Mechanical Science and Technology, 24, 865-868.
[14] Xu, L.-Y., Cao, Z.-Q., Zhao, P., Zhou, C. (2017). A new monocular vision measurement method to estimate 3D positions of objects on floor. International Journal of Automation and Computing, 14, 159-168.

[15] Yu, Q.-F., Shang, Y. (2009). Videometrics: Principles and Research. Beijing, China: Science Press.

[16] He, Z., Wang, B. (2003). Sub-pixel extraction algorithm using curve fitting method. Journal of Scientific Instrument, 24, 195-197.

[17] Chang, S.-T., Sun, Z.-Y., Zhang, Y.-Y., Zhu, W. (2014). Radiation measurement of small targets based on PSF. Optics and Precision Engineering, 22 (11), 2879-2887.

[18] Duan, Z.-Y., Wang, N., Zhao, W.-H. et al. (2016). Study on calibration method based on lattice calibration plate in vision measurement system. Acta Optica Sinica, 36 (5), 0515004.

[19] Fei, Z.-G, Xu, X.-J., Anthimos, G. (2016). Short-arc measurement and fitting based on the bidirectional prediction of observed data. Measurement Science and Technology, 27 (2), 025013.

Received August 11, 2017. Accepted March 20, 2018. 